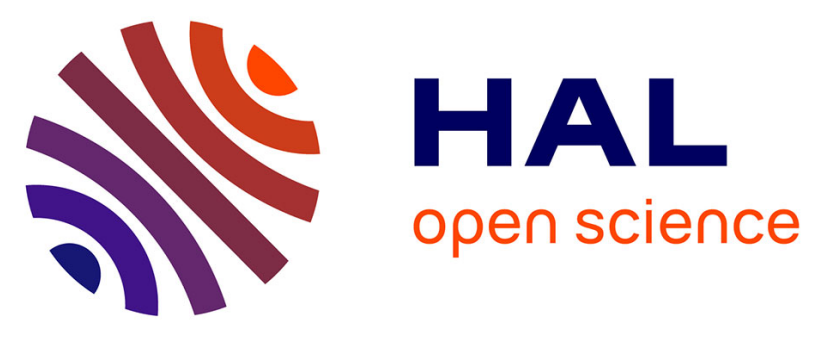

\title{
Severe axial and pelvifemoral muscle damage in immune-mediated necrotizing myopathy evaluated by whole-body MRI
}

Océane Landon-Cardinal, Cédi Koumako, Giulia Hardouin, Benjamin Granger, Harmen Reyngoudt, Jean-Marc Boisserie, Aude Rigolet, Baptiste

Hervier, Nicolas Champtiaux, Perrine Guillaume-Jugnot, et al.

\section{To cite this version:}

Océane Landon-Cardinal, Cédi Koumako, Giulia Hardouin, Benjamin Granger, Harmen Reyngoudt, et al.. Severe axial and pelvifemoral muscle damage in immune-mediated necrotizing myopathy evaluated by whole-body MRI. Seminars in Arthritis and Rheumatism, 2020, 50, pp.1437 - 1440. 10.1016/j.semarthrit.2020.02.009 . hal-03494062

\section{HAL Id: hal-03494062 https://hal.science/hal-03494062}

Submitted on 12 Jan 2022

HAL is a multi-disciplinary open access archive for the deposit and dissemination of scientific research documents, whether they are published or not. The documents may come from teaching and research institutions in France or abroad, or from public or private research centers.
L'archive ouverte pluridisciplinaire HAL, est destinée au dépôt et à la diffusion de documents scientifiques de niveau recherche, publiés ou non, émanant des établissements d'enseignement et de recherche français ou étrangers, des laboratoires publics ou privés. 


\title{
Severe axial and pelvifemoral muscle damage in immune-mediated necrotizing myopathy evaluated by whole-body MRI
}

\author{
Océane Landon-Cardinal ${ }^{\mathrm{a}, \mathrm{b}, \mathrm{c}, 1, *}$, Cédi Koumako ${ }^{\mathrm{d}, 1}$, Giulia Hardouin ${ }^{\mathrm{e}}$, Benjamin Granger ${ }^{\mathrm{e}}$, \\ Harmen Reyngoudt ${ }^{\mathrm{d}}$, Jean-Marc Boisserie ${ }^{\mathrm{d}}$, Aude Rigolet ${ }^{\mathrm{a}}$, Baptiste Hervier ${ }^{\mathrm{a}}$, \\ Nicolas Champtiaux ${ }^{\mathrm{a}}$, Perrine Guillaume-Jugnot ${ }^{\mathrm{a}}$, Mathieu Vautier ${ }^{\mathrm{a}}$, Olivier Benveniste ${ }^{\mathrm{a}}$, \\ Pierre G. Carlier ${ }^{\mathrm{d}}$, Yves Allenbach ${ }^{\mathrm{a}}$
}

\footnotetext{
a Department of Internal Medicine and Clinical Immunology and Inflammation-Immunopathology-Biotherapy Department (I2B), Pitié-Salpêtrière University Hospital, Assistance Publique-Hôpitaux de Paris, East Paris Neuromuscular Diseases Reference Center, Inserm U974, Sorbonne Université, Paris 6, Paris, France ${ }^{\mathrm{b}}$ Department of Medicine, Faculty of Medicine, University of Montreal, Montreal, Quebec, Canada

'Division of Rheumatology and Research Center, Centre Hospitalier de l'Université de Montréal, Montreal, Quebec, Canada

${ }^{\mathrm{d}}$ Laboratoire de RMN AIM \&' CEA, Centre d'Explorations Neuro-musculaires, Institut de Myologie, Paris, France

e Département de Biostatistiques, Santé Publique et Information Médicale, Groupe Hospitalier Pitié-Salpêtrière, Assistance Publique-Hôpitaux de Paris, Paris, France
}

\section{A R T I C L E I N F O}

\section{Keywords:}

Immune-mediated necrotizing myopathy Myositis

Myopathy

Whole-body MRI

Muscle imaging

\begin{abstract}
A B S T R A C T
Background: Our objective was to define the pattern and severity of muscle damage in immune-mediated necrotizing myopathy (IMNM) and its relationship with clinical and serological features.

Methods: IMNM patients with a whole-body MRI $(n=42)$ were included and compared to sporadic inclusionbody myositis (s-IBM) patients ( $n=60$ ). Fat replacement was estimated using the Mercuri score in 55 muscles. Overall lesion load was defined as the sum of all abnormal Mercuri scores (reported in \% maximal score) and lesion load quotient was defined as the overall lesion load divided by disease duration. Linear relationships between variables were assessed and multidimensional analysis was performed to define homogenous groups of patients.

Results: IMNM patients were aged $48.1 \pm 15.8$ years and had a disease duration of $9.8 \pm 8.1$ years. Most severely affected muscle groups were located in the pelvifemoral and lumbar region. Unsupervised analysis showed two subgroups of patients: one with mild lesion load $(15 \pm 10 \%, n=32 / 42)$ and another with severe lesion load $(60 \pm 10 \%, n=10 / 42: p<0.001)$ associated with a mean disease duration of $6.8 \pm 6.0$ years and $19.5 \pm$ 5.7 years, respectively $(p<0.0001)$. Correlational studies confirmed that disease duration was the most important predictor of muscle damage. Multivariate analyses demonstrated a more severe involvement in select muscle groups in females and seropositive patients.

No difference was found in overall lesion load quotient of IMNM compared to IBM ( $p=0.07)$ but with a distinct muscle pattern.

Conclusion: IMNM is associated with severe axial and pelvifemoral muscle damage. Disease duration is an important predictor of muscle damage. IMNM and s-IBM patients have a comparable damage burden.
\end{abstract}

Crown Copyright @ 2020 Published by Elsevier Inc. All rights reserved.

\footnotetext{
Abbreviations: IMNM, immune-mediated necrotizing myopathy; MRI, magnetic resonance imaging; WB-MRI, whole-body MRI; s-IBM, sporadic inclusion-body myositis; IST, immunosuppressive therapy

* Corresponding author at: Department of Internal Medicine and Clinical Immunology and Inflammation-Immunopathology-Biotherapy Department (I2B), Pitié-Salpêtrière University Hospital, Assistance Publique-Hôpitaux de Paris, East Paris Neuromuscular Diseases Reference Center, Inserm U974, Sorbonne Université, Paris 6 , Paris, France.

E-mail address: oceane.landon-cardinal@umontreal.ca (O. Landon-Cardinal).

1 These authors contributed equally to the manuscript.
}

\section{Introduction}

Immune-mediated necrotizing myopathy (IMNM) has been recently characterized as a distinct subset of autoimmune myopathy associated with severe muscle involvement [1-3]. Two autoantibodies have been associated with IMNM - anti-SRP and anti-HMGCR -, but IMNM can also be found in seronegative patients. A characteristic pattern of thigh muscle involvement and early muscle damage on magnetic resonance imaging (MRI) has been reported [3] and may explain poor muscle strength recovery observed in these patients at 4 years [3,4]. Interestingly, anti-SRP subset has been associated with 
a more severe thigh muscle involvement compared to anti-HMGCR, in a similar range as sporadic inclusion-body myositis (s-IBM) [3,5,6], another severely disabling muscle disease [7]. Muscle pattern involvement in other muscle groups has not been reported nor its relationship with serological status.

We therefore performed whole-body MRI in order to analyze (i) the pattern and the severity of muscle damage in IMNM and (ii) its relationship with serological status and the clinical course of the disease.

\section{Material and methods}

\section{Patients}

We performed a retrospective study including all consecutive patients with a diagnosis of IMNM according to the ENMC criteria [8] and who had a whole-body MRI (WB-MRI) between 2011 and 2017. Anti-HMGCR antibodies were identified using ELISA (Inova Diagnostics, Inc, San Diego, California) or addressable laser bead immunoassay (ALBIA, Rouen, France) [9]. Anti-SRP antibodies were identified using immunodot assays (Inova Diagnostics, Inc, San Diego, California).

Patients with s-IBM according to the Lloyd's criteria [10] and a WB-MRI were also included for comparison.

\section{Muscle MRI acquisition}

All MRI data were performed on a 3-T clinical system (Trio until December 2014 or Prisma from January 2015, Siemens Healthineers, Erlangen, Germany). The standardized protocol included coronal and axial T1-weighted Turbo Spin Echo images. In the coronal orientation, TR/TE was 600/9.1 ms on Trio and 800/9.1 ms on Prisma. In the axial orientation, TR/TE was 507/9.4 ms on Trio and 800/9.8 ms on Prisma. Slice thickness was $6 \mathrm{~mm}$. Thirty composed coronal images were generated. In the axial views, 174 slices were obtained (Fig. 1).

\section{Image evaluation}

Image analysis was performed by two imaging specialists (CK and PC) and 55 muscles were evaluated bilaterally. Fat replacement was estimated in $\mathrm{T} 1$ sequence using the Mercuri score (1=normal; $2=$ mild involvement and fat replacement $<30 \%, 3=$ moderate involvement, 30-60\%; 4=severe involvement, >60\%) (Appendix A).

\section{Muscle damage scoring}

Overall lesion load was defined as the sum of all abnormal Mercuri scores $(\geq 2)$ evaluated in each muscle (reported in percentage from minimum $0 \%$ to maximum $100 \%$ ) and lesion load quotient was defined as the overall lesion load divided by the disease duration (years) at WB-MRI. Mercuri scores was subsequently adjusted for the relative muscle mass of each muscles (Appendix-Fig. A).

\section{Statistical analysis}

Qualitative data was reported as percentages and quantitative data was reported as means \pm standard deviation (SD). Principal Component Analysis and Ascending Hierarchical Classification were performed to assess the linear relationships between variables and define homogenous groups of patients. Multivariate linear regression was subsequently performed to evaluate predictors of muscle damage. Statistical analyses were performed using R software 3.4.2.

\section{Ethic statements}

Written informed consent from each study patient and approval by local Ethics Committee (CPP Ile De France VI (2013-12-19), CCTIRS $\left(\mathrm{N}^{\circ} 14.323\right)$ and $\mathrm{CNIL}$ (915139)) was obtained to use medical information recorded in the myositis database for scientific purposes.

\section{Results}

Patients

Forty-two IMNM patients (anti-HMGCR, $n=25$; anti-SRP, $n=12$ and seronegative, $n=5$ ) and $60 \mathrm{~s}$-IBM patients were included. Patients' characteristics are displayed in Table 1. Disease duration at WB-MRI was similar in IMNM and s-IBM $(9.8 \pm 8.1$ vs $8.9 \pm 5.2$ years, respectively).

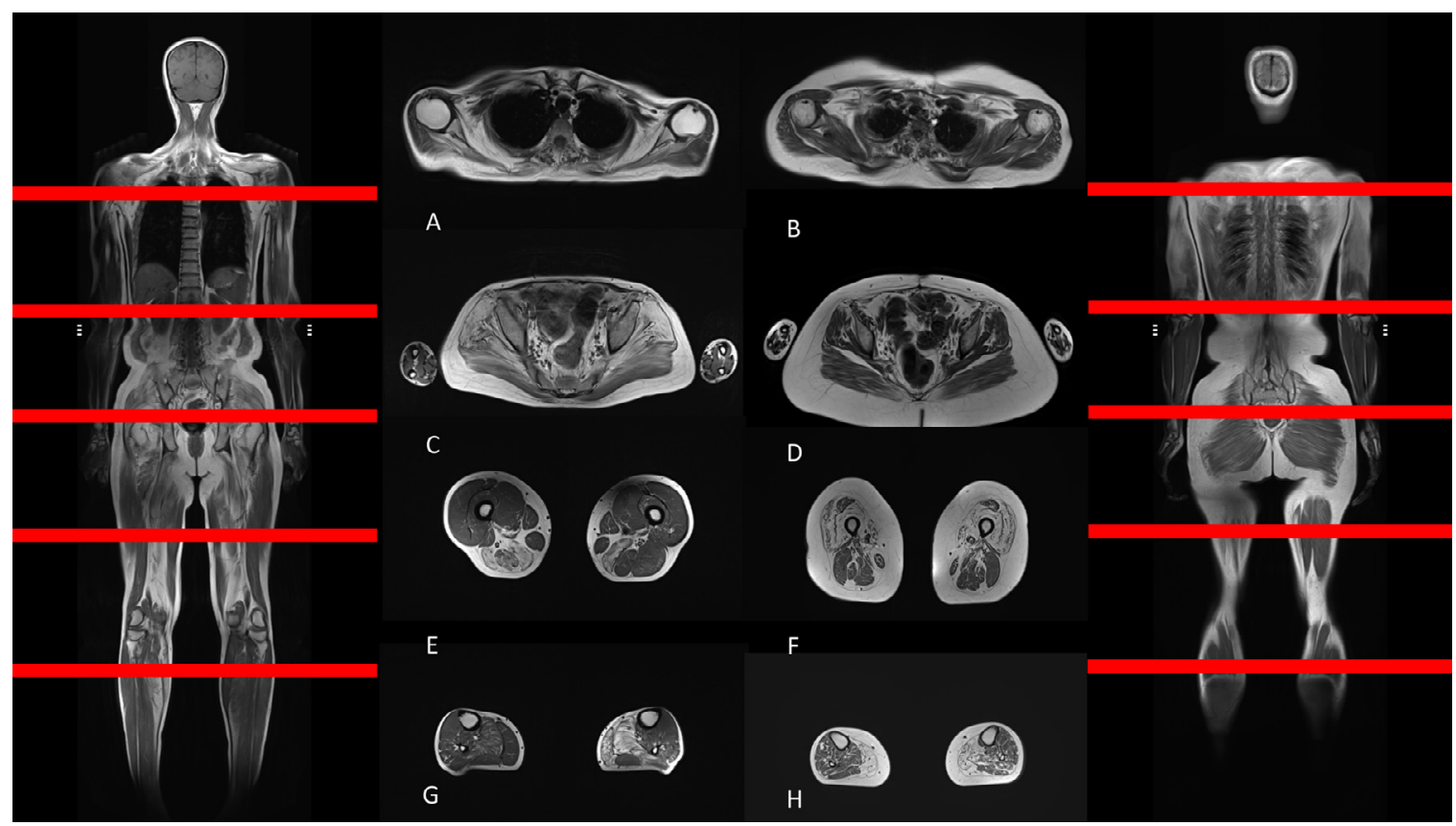

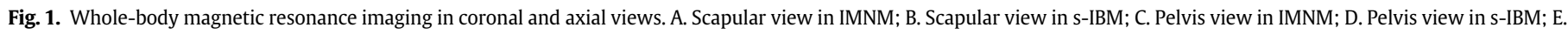
Thigh view in IMNM; F. Thigh view in s-IBM; G. Leg view in IMNM; H. Leg view in s-IBM. 
Table 1.

Patients characteristics at WB-MRI

\begin{tabular}{lll}
\hline & $\operatorname{IMNM}(n=42)$ & s-IBM $(n=60)$ \\
\hline Female, \% & 63 & 47 \\
Age at WB-MRI, yrs & $48.1 \pm 15.8$ & $66.3 \pm 8.3$ \\
Disease duration, yrs & $9.8 \pm 8.1$ & $8.9 \pm 5.2$ \\
Treatment initiation delay, yrs & $4.8 \pm 7.1$ & NA \\
Caucasian, \% & 64 & 98 \\
Anti-HMGCR, \% & 60 & NA \\
Anti-SRP, \% & 28 & NA \\
Seronegative, \% & 12 & NA \\
Mean cumulative prednisone dose, mg & 16,959 & NA \\
Cancer-associated malignancy, \% & 3 & NA \\
Mean CK level, IU/L & $606 \pm 580$ & NA \\
\hline
\end{tabular}

yrs = years; NA = not applicable

Comparable overall muscle damage severity in IMNM vs s-IBM but with distinct patterns

No difference in overall lesion load was observed in IMNM compared to s-IBM ( $26 \pm 22 \%$ vs $22 \pm 14 \%, p=0.3$ ), nor in lesion load quotient (lesion load divided by disease duration) ( $14 \pm 15$ vs $9 \pm 8, p=0.07$ ), even when adjusting for the relative muscle mass of each muscles (Appendix A). Nevertheless, as expected, the muscle damage pattern was different in these two myopathies. Most severely affected muscle groups in IMNM (mean Mercuri \pm SD) were located in the pelvifemoral muscle groups (gluteus minimus: $2.7 \pm 1.1$ and gluteus medius: $2.6 \pm 1.1$ ), lumbar region (lumbar extensors: $2.7 \pm 1.1$ ) and shoulder region (subscapularis: $2.7 \pm$ 1.1) (all $p<0.0001$ compared to s-IBM, Figs. 1 and 2). On the opposite, in s-IBM patients' muscles were preferentially affected in the anterior muscle thigh compartment (vastus lateralis: $2.7 \pm 0.8$, vastus medialis: $2.6 \pm 0.8$ and vastus intermedialis: $2.6 \pm 0.8$ ) and in the calf muscles (gastrocnemius medial: $2.8 \pm 1.1$ ) (all $p<0.0001$ compared to IMNM, Figs. 1 and 2).
Muscle damage severity is dependent on disease duration

Unsupervised analysis of the IMNM subset showed two groups of patients: one with a mild lesion load $(15 \pm 10 \%, n=32 / 42)$ and another with a severe lesion load $(60 \pm 10 \%, n=10 / 42$ : $p<0.001)$. Disease duration at WB-MRI was $6.8 \pm 6.0$ years compared to $19.5 \pm 5.7$ years $(p<0.0001)$ and time from symptom onset to treatment initiation was longer in the latter group ( $11.5 \pm 9.2$ vs $2.5 \pm 4.6$ years, $p<0.0001)$, respectively. Correlational studies confirmed that disease duration was the most important predictor of muscle damage (AppendixTable A).

\section{Demographic and serological status impact on muscle damage in IMNM}

Multivariate analyses - adjusted for disease duration, age at first symptoms and time from symptom onset to treatment initiation demonstrated a more severe involvement of the gluteus maximus $(p=0.04)$ and the great adductor $(p=0.04)$ in seropositive (antiHMGCR and anti-SRP) patients, and a more severe involvement in the great adductor $(p=0.02)$ and the vastus lateralis $(p=0.02)$ in females (Appendix-Table B).

\section{Discussion}

To our knowledge, this is the first study reporting the pattern of muscle involvement and damage in IMNM compared to s-IBM patients using WB-MRI. We observed that disease duration appears to be most important predictor of muscle damage and that gender and serological status may influence muscle damage pattern. Strikingly, overall muscle damage severity was comparable in IMNM and s-IBM but, as expected, with distinct muscle groups involvement.

Early muscle thigh damage has previously been reported with a more severe involvement in anti-SRP compared to anti-HMGCR

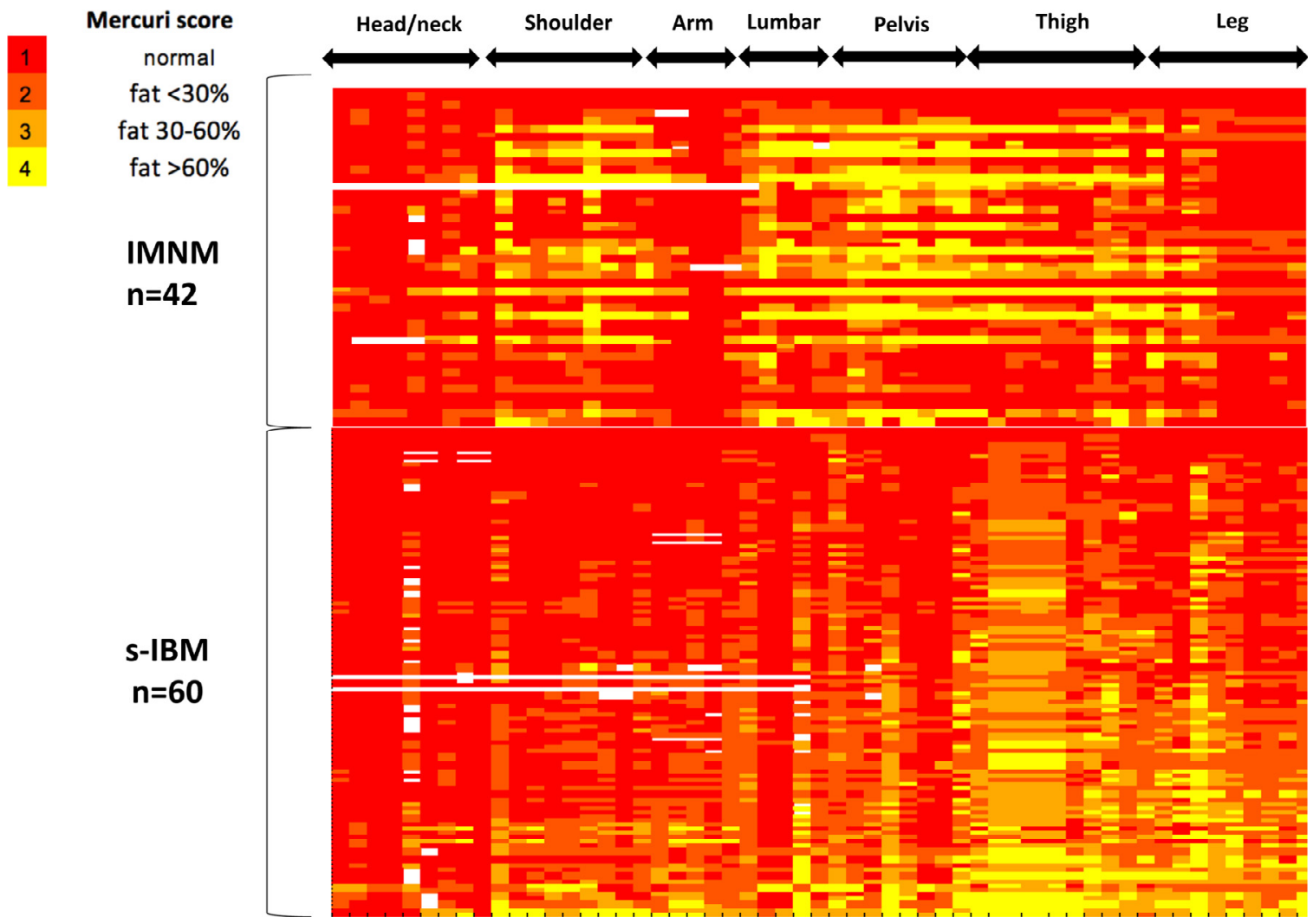

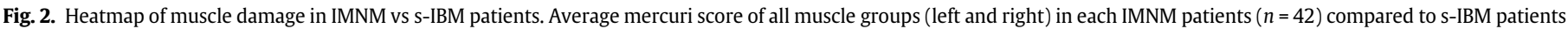
$(n=60)$. 
IMNM [3,5]. In this study, WB-MRI evaluation has permitted to assess the involvement of other muscles and our data confirm the pelvifemoral muscle group is predominantly affected in IMNM, nevertheless, selective involvement of lumbar and shoulder muscle regions was also observed. Interestingly, scapular winging has been reported in IMNM, while this feature is usually considered unusual in other autoimmune myopathies [11]. Of note, the presence of scapular winging has previously been reported in up to a third of patients with a limb-girdle muscular dystrophy (LGMD)-like presentation of anti-HMGCR myopathy [12].

A more severe involvement in some muscle groups was found in seropositive patients but we did not observe that anti-SRP patients had more severe involvement than anti-HMGCR. This may be due to a lack of statistical power related to our small sample size or longer disease duration in our cohort [12]. Our data also suggest female patients may have a more severe involvement in some muscles which is a novel finding in IMNM.

In this study, we report for the first time the overall damage burden in IMNM compared to s-IBM using WB-MRI and adjusting for the relative muscle mass of each muscle while taking into account the disease duration, which is a major predictor of muscle damage. Surprisingly, no difference was observed in the damage burden of IMNM compared to s-IBM even though the former is a treatment-responsive myopathy while the latter is a severely disabling muscle disease refractory to standard IST [7] and associated with an annual thigh fat replacement progression of 3\% [13]. This finding may indirectly reflect the aggressiveness of the IMNM subset and emphasize the importance of differentiating disease activity vs damage, using MRI sequences that detect both the inflammation and damage, that are relevant for assessing muscle weakness in IMNM patients.

We acknowledge that fatty replacement may be influenced by many factors, in addition to unsuppressed inflammation, including corticosteroids, aging and inactivity. Prospective longitudinal WB-MRIs should be explored to clarify their impact on muscle damage. Our data also underline that autoimmune myopathies display a selective pattern of muscle involvement and its characterization is of importance to refine clinical assessment of myositis patients for diagnosis purposes [6] but also follow-up after therapeutic interventions. The pattern of involvement reported herein is consistent with the clinical description of IMNM and s-IBM where a proximal lower-limb dominant and anterior thigh/leg involvement has been reported, respectively [14,15].

We acknowledge that many of our patients had a prolonged disease duration prior to diagnosis and treatment initiation, and patients referred to our center may represent a subset of more severe and refractory patients as thirty-eight percent of the patients included in this study presented a chronic "dystrophy-like" presentation of IMNM $[1,12]$. These findings may not be generalizable to the whole spectrum of IMNM. Nonetheless, our results underline the high damage burden associated with IMNM. The potential of MRI as a biomarker of damage progression remain to be explored longitudinally in IMNM patients.

\section{Conclusion}

IMNM is associated with a severe muscle damage in the axial and pelvifemoral muscle groups. Disease duration is an important predictor of muscle damage and gender and serological status may also influence muscle damage pattern. IMNM and s-IBM patients have comparable overall damage burden but with a distinct muscle pattern.

\section{Declaration of Competing Interest}

None.

\section{Author contribution}

Conceptualization and design: OLC, BG, OB, PC, YA.
Acquisition of data: OLC, CK, HR, JMB, AR, BH, NC, PGJ, MV, OB, PC and YA.

Analysis and interpretation: OLC, CK, GH, BG, HR, JMB, OB, PC and YA.

Critical revision of the manuscript for important intellectual content: OLC, CK, GH, BG, HR, JMB, AR, BH, NC, PGJ, MV, OB, PC and YA.

\section{Acknowledgment}

None.

\section{Funding}

This research did not receive any specific grant from funding agencies in the public, commercial, or not-for-profit sectors.

\section{Disclosure}

Dr. Landon-Cardinal is the recipient of Clinical Fellowship awards from the Université de Montréal Rheumatology Program - Abbvie Educational Grant and the Association des médecins rhumatologues du Québec - Visithan-Khy Educational Grant. CK, GH, BG, HR, JMB, $\mathrm{AR}, \mathrm{BH}, \mathrm{NC}, \mathrm{PGJ}, \mathrm{MV}, \mathrm{OB}, \mathrm{PC}$ and YA report no disclosure.

\section{Supplementary materials}

Supplementary material associated with this article can be found in the online version at doi:10.1016/j.semarthrit.2020.02.009.

\section{References}

[1] Allenbach Y, Drouot L, Rigolet A, Charuel JL, Jouen F, Romero NB, et al. AntiHMGCR autoantibodies in European patients with autoimmune necrotizing myopathies: inconstant exposure to statin. Medicine 2014;93(3):150-7.

[2] Watanabe Y, Uruha A, Suzuki S, Nakahara J, Hamanaka K, Takayama K, et al. Clinical features and prognosis in anti-SRP and anti-HMGCR necrotising myopathy. J Neurol Neurosurg Psychiatry 2016;87(10):1038-44

[3] Pinal-Fernandez I, Casal-Dominguez M, Carrino JA, Lahouti AH, Basharat P, Albayda J, et al. Thigh muscle MRI in immune-mediated necrotising myopathy: extensive oedema, early muscle damage and role of anti-SRP autoantibodies as a marker of severity. Ann Rheum Dis 2017;76(4):681-7.

[4] Tiniakou E, Pinal-Fernandez I, Lloyd TE, Albayda J, Paik J, Werner JL, et al. More severe disease and slower recovery in younger patients with anti-3-hydroxy-3methylglutaryl-coenzyme A reductase-associated autoimmune myopathy. Rheumatology 2017;56(5):787-94

[5] Zheng Y, Liu L, Wang L, Xiao J, Wang Z, Lv H, et al. Magnetic resonance imaging changes of thigh muscles in myopathy with antibodies to signal recognition particle. Rheumatol Oxf Engl 2015;54(6):1017-24

[6] Day JA, Bajic N, Gentili S, Patel S, Limaye V. Radiographic patterns of muscle involvement in the idiopathic inflammatory myopathies. Muscle Nerve 2019:60(5):549-57.

[7] Benveniste O, Guiguet M, Freebody J, Dubourg O, Squier W, Maisonobe T, et al. Long-term observational study of sporadic inclusion body myositis. Brain 2011;134(11):3176-84.

[8] Allenbach Y, Mammen AL, Benveniste O, Stenzel W, Allenbach Y, Amato A, et al 224th ENMC international workshop. Neuromuscul Disord 2018;28(1):87-99.

[9] Drouot L, Allenbach Y, Jouen F, Charuel J-L, Martinet J, Meyer A, et al. Exploring necrotizing autoimmune myopathies with a novel immunoassay for anti-3hydroxy-3-methyl-glutaryl-CoA reductase autoantibodies. Arthritis Res Ther 2014;16(1):R39.

[10] Lloyd TE, Mammen AL, Amato AA, Weiss MD, Needham M, Greenberg SA. Evaluation and construction of diagnostic criteria for inclusion body myositis. Neurology 2014;83(5):426-33.

[11] Mammen AL. Which nonautoimmune myopathies are most frequently misdiagnosed as myositis? Curr Opin Rheumatol 2017;29(6):618-22.

[12] Mohassel P, Landon-Cardinal O, Foley AR, Donkervoort S, Pak KS, Wahl C, et al. Anti-HMGCR myopathy may resemble limb-girdle muscular dystrophy. Neurol Neuroimmunol Neuroinflammation 2019;6(1):e523.

[13] Morrow JM, Sinclair CDJ, Fischmann A, Machado PM, Reilly MM, Yousry TA, et al. MRI biomarker assessment of neuromuscular disease progression: a prospective observational cohort study. Lancet Neurol 2016;15(1):65-77.

[14] Mariampillai K, Granger B, Amelin D, Guiguet M, Hachulla E, Maurier F, et al Development of a new classification system for idiopathic inflammatory myopathies based on clinical manifestations and myositis-specific autoantibodies. JAMA Neurol 2018;75(12):1528-37.

[15] Greenberg SA. Inclusion body myositis: clinical features and pathogenesis. Nat Rev Rheumatol 2019;15(5):257-72. 